\title{
Evaluación de la eficiencia en disipación de energía en estructuras hidráulicas construidas con gaviones y material reciclado (neumático usado) mediante modelamiento físico a escala reducida \\ Evaluation of energy dissipation efficiency in hydraulic structures built with gabions and recycled material (used tire) by means of physical modeling on a reduced scale
}

\author{
Luis Efrén Ayala-Rojas ${ }^{1}$, Alejandro Franco-Rojas ${ }^{2 a}$, Edgar Alexander Padilla-González ${ }^{2 b}$ \\ ${ }^{1}$ Centro de Investigación en Riesgos de Obras Civiles (Ciroc), Universidad de La Salle, Colombia. \\ Correo electrónico: layalar@unisall.edu.co \\ ${ }^{2}$ Grupo de Investigación en Desarrollo Tecnológico (Indetec), Universidad de La Salle, Colombia. \\ Correo electrónicos: ${ }^{a}$ afrancor@ unisalle.edu.co, ${ }^{\mathrm{b}}$ edpadilla@ unisalle.edu.co
}

Recibido: 10 febrero, 2019. Aceptado: 22 septiembre, 2018. Versión final: 30 diciembre, 2019.

\begin{abstract}
Resumen
Flujos con exceso de energía cinética producen alta velocidad, sobrepresiones y procesos erosivos, en este sentido los disipadores de energía son estructuras hidráulicas que minimizan este efecto. Dichas obras suelen construirse en concreto convencional, dejando de lado materiales alternativos como neumático usado y gaviones. Fueron evaluadas tres estructuras de disipación de energía: un canal escalonado ubicado en la vía Bogotá -Villavicencio, un pozo de alcantarillado pluvial con estructura de caída escalonada ubicado en el municipio Acacias (Meta) y cuatro vertederos de caída libre construidos en concreto, posicionados sobre el río Fucha en Bogotá. El modelamiento a escala reducida muestra que, al incorporar gaviones y neumático usado, se conserva el tipo de flujo (nappe flow), su funcionalidad, la disipación de energía supera el $92 \%$, adicionalmente reduce costos y aporta a conservar la estabilidad morfológica del cauce, particularmente, permiten concentrar el flujo por el canal principal y suavizar los cambios de dirección.
\end{abstract}

Palabras clave: disipación de energía; gaviones; modelamiento físico; neumático usado.

\begin{abstract}
Flows with excess of kinetic energy produce high speed, overpressures and erosive processes, in this sense the energy dissipaters are hydraulic structures that minimize this effect. These works are usually built in conventional concrete, leaving aside alternative materials such as used tire and gabions. Three energy dissipation structures were evaluated: a stepped channel located on the Bogota -Villavicencio road, a storm sewer well with stepped fall structure located in the municipality of Acacias (Meta) and four free-fall spillway weir built in concrete, positioned on the Fucha river in Bogotá. Modeling on a reduced scale shows that, when incorporating gabions and used tires, the flow type (nappe flow) is conserved, its functionality, energy dissipation is greater than $92 \%$, additionally reduces costs and contributes
\end{abstract}

ISSN impreso: 1657 - 4583. ISSN en línea: 2145 - 8456, CC BY-ND 4.0 (c) (i) $\ominus$

L. Ayala-Rojas, A. Franco-Rojas, E. A. Padilla-González, "Evaluación de la eficiencia en disipación de energía en estructuras hidráulicas construidas con gaviones y material reciclado (neumático usado) mediante modelamiento físico a escala reducida," Rev. UIS Ing., vol. 19, no. 1, pp. 143-154, 2020. doi: 10.18273/revuin.v19n1-2020014 
to the conservation the morphological stability of the channels, particularly, they allow concentrating the flow through the main channel and smoothing the changes of direction.

Keywords: dissipation of energy; gabions; physical modeling; used tire.

\section{Introducción}

La mayoría de obras hidráulicas que operan a flujo libre, operan sometidas a fuertes corrientes, cuya energía deteriora progresivamente los materiales, reduciendo su vida útil e incluso la funcionalidad [1]. Aunado a ello, el costo de estructuras convencionales minimiza las posibilidades de renovación, dando lugar a daño en malla vial, colapso de sistemas de alcantarillado e incremento de fenómenos amenazantes sobre la población y sus bienes. El uso de materiales alternativos, como neumático usado y gaviones, busca innovar en estructuras hidráulicas eficientes, económicas y amigables al medio ambiente, sin que ello signifique una pérdida de funcionalidad o un riesgo sobre las obras de infraestructura y la población.

Para verificar el cumplimiento de su principal función que es la disipación de energía, es decir, que sea similar o mejor que las estructuras revestidas en concreto, es preciso comparar su operación bajo condiciones controladas similares, exigiendo el modelamiento físico a escala reducida, de tal forma que se respete su geometría, tipo de flujo y cantidad de caudal que fluye por la estructura, lo cual se logra con el uso de principios de similitud:

- Similitud geométrica. Dos objetos son geométricamente similares si las razones de sus dimensiones correspondientes son iguales, esto permite dimensionar todas las características geométricas del modelo a una misma escala, asegurando la similitud de forma [2], es decir, que el modelo y el prototipo son geométricamente similares si y solo si todas las dimensiones del cuerpo en cada una de los tres ejes coordenados, se relacionan mediante la misma escala de longitud [4].

- Similitud cinemática. Dos movimientos son cinematicamente similares cuando a) los patrones o trayectorias del movimiento son geométricamente similares y b) las razones de las velocidades de las partículas involucradas en los dos movimientos son iguales [2], lo anterior, obliga a que modelo y prototipo tengan una relación de escala de tiempos, de tal forma, que todos los caudales guardan la misma relación entre modelo y prototipo.

- Similitud dinámica. Dos movimientos son dinamicamente similares si a) la razón de las masas de los objetos involucrados son iguales y b) las razones de las fuerzas que afectan el movimiento son iguales [2]. Cumplir con la similitud dinámica implica previamente el cumplimiento de la similitud cinética y que todas las fuerzas dinámicas: inerciales, internas, gravitacionales, elásticas, viscosas, entre otras. están en la misma relación o escala en los dos sistemas (modelo y prototipo), condición inalcanzable. Siendo necesario identificar las fuerzas predominantes en el modelo y garantizar que los parámetros adimensionales que representan dichas fuerzas sean iguales tanto en el modelo como en el prototipo. En el caso de flujos a superficie libre, la fuerza predominante es la aceleración de la gravedad, por tanto, el parámetro adimensional representativo será el número de Froude (ver Ecuación (1)).

Donde:

$$
\mathrm{F}=\frac{\mathrm{V}}{\sqrt{\mathrm{gD}}}
$$

F: Número de Froude que relaciona las fuerzas inerciales y las fuerzas gravitacionales (adimensional)

$\mathrm{V}$ : Velocidad $(\mathrm{m} / \mathrm{s})$

g: Aceleración de la gravedad $\left(9.806 \mathrm{~m} / \mathrm{s}^{2}\right)$

D: Profundidad hidráulica que relaciona el área y ancho superficial del flujo (m)

Se evaluaron tres estructuras: vertederos en gaviones, un canal escalonado y un pozo de alcantarillado con caída hidráulica, estos dos últimos revestidos en neumático usado. Los resultados permitieron comparar la eficiencia hidráulica, disipación de energía y el comportamiento del flujo (líneas de flujo y turbulencia) en modelos a escala, respecto a obras similares construidas con materiales convencionales, demostrando en todos los casos, mayor capacidad de disipación de energía y corrección de patrones de flujo indeseables.

Implementar estos materiales en futuros proyectos de ingeniería o en reposición y mantenimiento de los mismos, aportará a minimizar el vertimiento de llantas usadas cuyos componentes no biodegradables tardan aproximadamente 100 años para su degradación total, además, el logro de sistemas que se incorporan fácilmente al paisaje, como es el caso de los vertederos en gaviones. 


\section{Fundamentos teóricos}

Los disipadores de energía son estructuras hidráulicas, utilizadas para disminuir exceso de energía cinética del flujo, expresada en altas velocidades, que, al intentarse reducir producen grandes presiones y la aparición de saltos hidráulicos e impactos del agua sobre el material expuesto, logrando con ello prevenir erosión aguas abajo.

Las estructuras más utilizadas para disipación de energía son: canales escalonados, canales con pantallas deflectoras, vertederos de caída libre y pozos de amortiguación en canales de alta pendiente; sistemas que en general, dan lugar a aparición de alta turbulencia y resaltos hidráulicos. Para cuantificar la energía cinética del flujo en un canal se utiliza la ecuación de energía específica, E, definida como la energía por unidad de peso del agua que fluye a través de la sección, medida respecto al fondo del canal (ver Ecuación (2)). De esta manera, la eficiencia del sistema de disipación resulta de la relación adimensional entre la energía especifica antes y después de la estructura [5].

Donde:

$$
\mathrm{E}=\mathrm{Y} \cos \theta+\alpha \frac{\mathrm{V}^{2}}{2 \mathrm{~g}}
$$

Y: Altura de lámina de agua medida perpendicular al fondo $(\mathrm{m})$

$\mathrm{V}$ : Velocidad $(\mathrm{m} / \mathrm{s})$

$\mathrm{g}$ : Aceleración de la gravedad $\left(9.806 \mathrm{~m} / \mathrm{s}^{2}\right)$

$\theta$ : Pendiente longitudinal del canal (grados)

$\alpha$ : Coeficiente de Coriolis (1.0)

Particularmente, los resaltos hidráulicos tienen una alta capacidad de disipación de energía, resultante del cambio brusco de flujo supercrítico (rápido) a flujo subcrítico (lento), que a su vez se manifiesta en gran turbulencia y

aumento de la profundidad hacia aguas abajo, según se muestra en la Figura 1.

Los disipadores de energía de caída libre reducen la energía a través del impacto generado por el choque entre el agua que cae y la cama de agua después de la caída, así como por el resalto hidráulico que se forma aguas abajo de la zona de impacto. De acuerdo con los Artículos 155 y 159 de la Resolución 0330 de 2017 [6], este tipo de disipadores se recomiendan para caídas de hasta $7 \mathrm{~m} \mathrm{y}$ descargas a canales, cuerpos de agua o a cielo abierto donde se requiera reducir la velocidad y evitar la socavación del terreno. Por su parte, en los canales escalonados la disipación de energía se da en cada escalón y su eficiencia de disipación está en función de la altura del escalón y el número de escalones que componen el canal, producto del rompimiento y mezcla del chorro, y por la formación de un resalto hidráulico en el escalón. Este tipo de estructuras según el Capítulo 4 del manual del INVIAS [7], buscan asegurar una adecuada entrega evitando problemas de socavación y se deben diseñar para periodos de retorno de 10 años.

En sistemas escalonados pueden ocurrir dos tipos de régimen mostrados en la Figura 2. flujo saltante (Nappe flow) caracterizado por sucesión de chorros en caída libre que chocan en el siguiente escalón y ocasionan un resalto hidráulico, o flujo rasante (Skimming flow) caracterizado porque el agua fluye sobre los escalones a manera de una corriente estable sobre una superficie altamente rugosa semejante a un "flujo espumoso" [8].

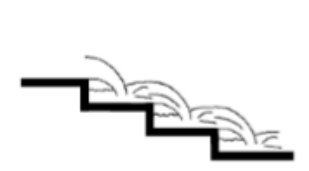

(a)

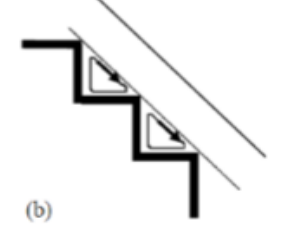

Figura 2. Tipo de flujo sobre vertederos escalonados. (a) Flujo saltante (b) Flujo rasante. Fuente: [9].

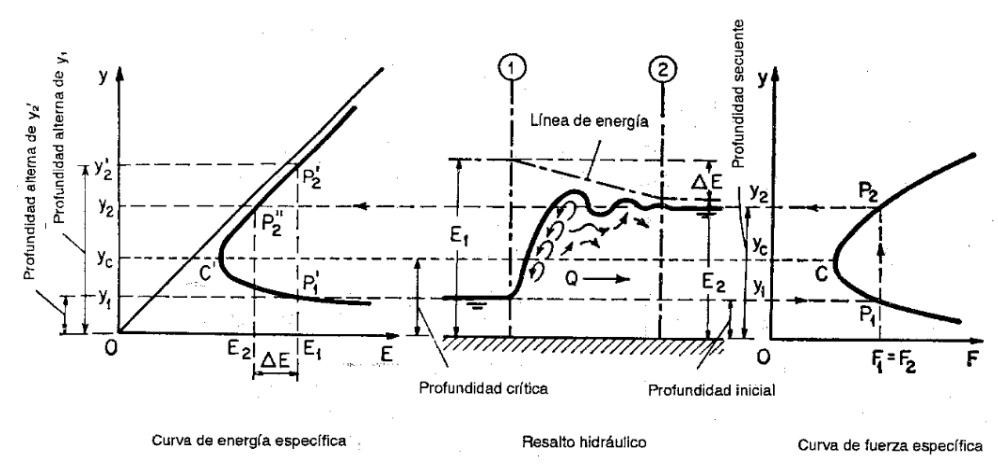

Figura 1. Curvas de energía específica y fuerza específica en un resalto hidráulico. Fuente: [3]. 
En los dos casos, se logra la disipación de energía y la incorporación de aire en cada escalón, redundando en el mejoramiento de la calidad físico-química del agua.

El modelamiento físico de estructuras hidráulicas exige el cumplimiento de los principios de similitud geométrica, cinemática y dinámica, que se consigue, según se explicó, cuando la razón de las masas, las fuerzas y velocidades de las particulas involucradas en ambos sistemas (prototipo y modelo) son iguales [2], y en caso de flujos a superficie libre corresponde al número de Froude que relaciona fuerzas de inercia y fuerzas de resistencia.

\section{Metodología}

En las instalaciones del laboratorio de hidráulica de la Universidad de La Salle se construyeron modelos a escala reducida de estructuras existentes, como son:

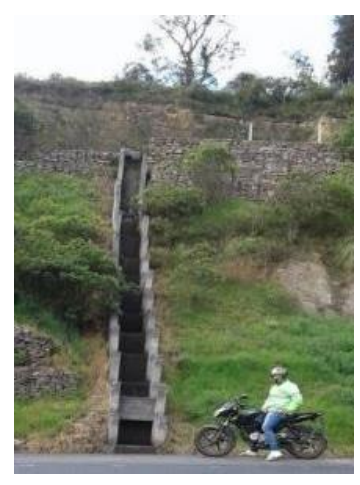

Figura 3. Estructuras de disipación (prototipo) con canal escalonado. Fuente: [9].

- Un canal escalonado ubicado en el km 11 de la vía Bogotá -Villavicencio, con pendiente del $82 \%$, ancho de $0.80 \mathrm{~m}$ y altura total de $13.7 \mathrm{~m}$ (ver Figura 3 ). Modelo desarrollado por [9].

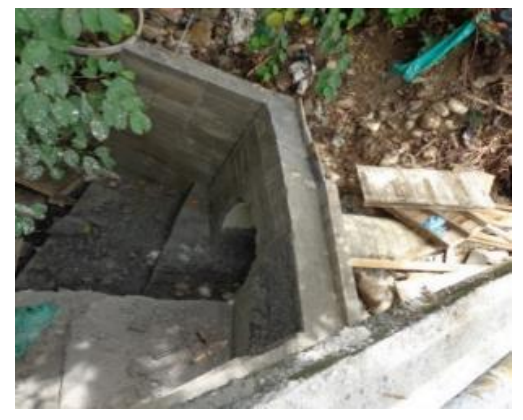

Figura 4. Estructura de disipación en la descarga de un pozo pluvial. Fuente: [10].
- Pozo de alcantarillado pluvial P144 con estructura de caída escalonada, ubicado sobre la calle 13 en el municipio Acacias (Meta), con longitud total de $6.80 \mathrm{~m}$, caída de $2.50 \mathrm{~m}$ y tubería de llegada con diámetro de 48” (ver Figura 4). Modelo desarrollado por [9].

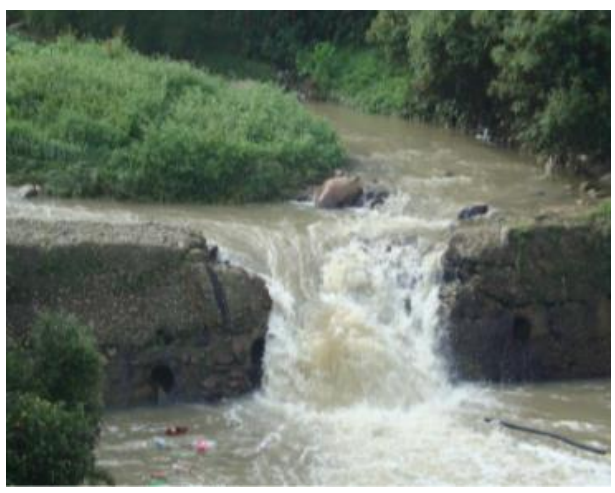

Figura 5. Estructuras de disipación (prototipo) con vertedero de caída libre. Fuente: [11].

- Cuatro vertederos de caída libre construidos en concreto, posicionados dentro del cauce del río Fucha, en la ciudad de Bogotá entre carreras quinta y sexta. Modelo desarrollado por [10].

Inicialmente, se realizó visitas de campo para medir la geometría e identificar detalles constructivos y operativos. Con el río Fucha se tuvo acceso a planos de levantamiento topográfico tanto del lecho del río como de las estructuras, permitiendo determinar las dimensiones de cada modelo con escalas geométricas mostradas en la Tabla 1.

Tabla 1. Escala geométrica y dimensiones de cada modelo hidráulico

\begin{tabular}{|l|l|l|l|l|}
\hline \multirow{2}{*}{ Estructura } & \multicolumn{2}{|l|}{ Escala del modelo } & \multicolumn{2}{l|}{ Caudal (1/s) } \\
\cline { 2 - 5 } & Horizontal & Vertical & Prototipo & Modelo \\
\hline $\begin{array}{l}\text { Canal } \\
\text { escalonado }\end{array}$ & $1: 2.5$ & $1: 10$ & 58.4 & 0.82 \\
\hline $\begin{array}{l}\text { Pozo con } \\
\text { estructura de } \\
\text { caída }\end{array}$ & $1: 3$ & $1: 2$ & 34.3 & 2.20 \\
\hline $\begin{array}{l}\text { Vertederos } \\
\text { en gaviones }\end{array}$ & $1: 100$ & $1: 20$ & 820 & 0.09 \\
\hline
\end{tabular}

Fuente: elaboración propia.

En todos los casos se construyeron y simularon dos modelos, uno con materiales convencionales (concreto) y otro con materiales alternativos. En el caso del canal escalonado y el pozo de alcantarillado pluvial la superficie se revistió con neumático usado, con piso Power Deck negro, que es una tableta fabricada con ripio (grano de caucho provenientes del reciclaje de neumático 
usados); mientras que en los vertederos se utilizaron gaviones fabricados con grava fina y malla plástica (ver Figura 6).

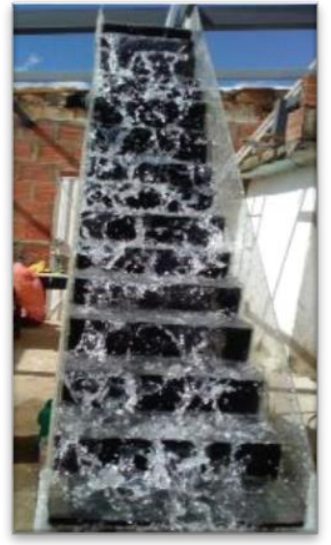

(a)

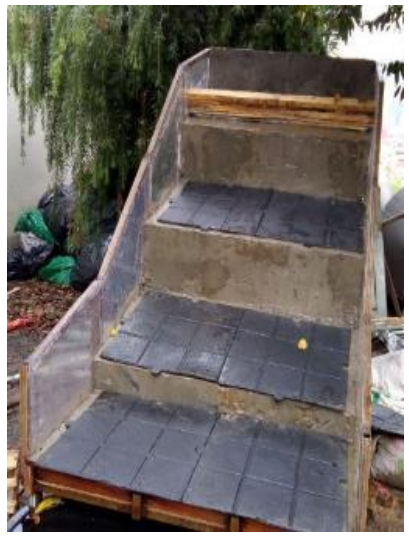

(b)

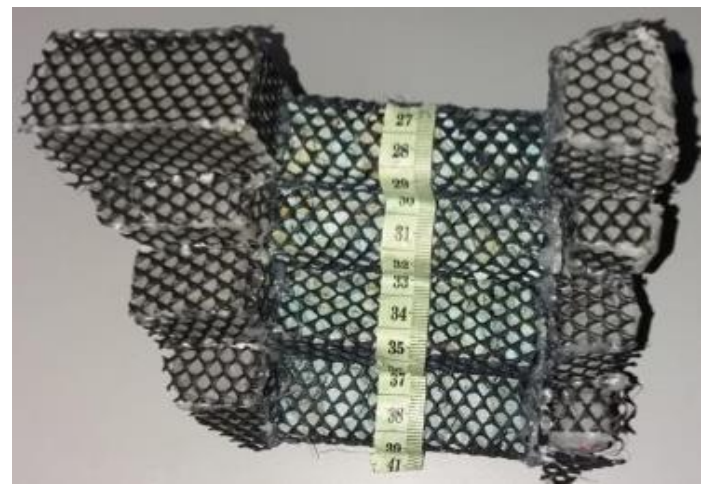

(c)

Figura 6. (a) Modelo hidráulico a escala reducida para el canal escalonado, (b) pozo pluvial con estructura de caída, (c) vertedero en gaviones. Fuente: elaboración propia a partir de [9] y [10].

Los datos recolectados incluyeron el aforo de caudal por el método gravimétrico, medición de altura de lámina de agua antes, sobre y después de la estructura de disipación, así como registro fotográfico de las corrientes de flujo previa aplicación de azul de metileno. Con estos datos se evaluó la energía específica, eficiencia y las líneas de flujo.

Teniendo en cuenta la naturaleza y función del sistema del cual hacen parte las estructura analizadas, así como la disponibilidad de información, fue necesario determinar los caudales de operación siguiendo métodos particulares en cada caso, según se detalla a continuación:

- El canal escalonado revestido con neumático, localizado en la vía Bogotá -Villavicencio permite el manejo de aguas de escorrentía, siendo necesario utilizar un modelo lluvia-escorrentía. Se seleccionó el método racional, recomendado para superficies de drenaje menores a 80 ha [6], el cual permite establecer el caudal de diseño a partir de parámetros simples como el área de drenaje, intensidad de la precipitación y el coeficiente de escorrentía, que, a su vez, es función del tipo de terreno y cobertura vegetal (ver Ecuación (3)).

$$
\mathrm{Q}_{\mathrm{r}}=2.78 * \mathrm{C} * \mathrm{I} * \mathrm{~A}
$$

Donde:

$\mathrm{Q}_{\mathrm{r}}$ : caudal superficial o real $(\mathrm{l} / \mathrm{s})$

C: coeficiente de escorrentía (0.88)

I: intensidad promedio de la lluvia $(62.79 \mathrm{~mm} / \mathrm{hr})$, según registros de la estación Chipaque (3502005)

A: área de drenaje ( $0.38 \mathrm{ha})$

- Para el pozo de alcantarillado pluvial P144, se determinó el caudal de diseño mediante aportes de áreas aferentes a la red de alcantarillado para un periodo de retorno de 1 en 50 años, considerando intensidad de $114,18 \mathrm{~mm} / \mathrm{hr}$, según reporte de EDESA S.A., complementados con aforos realizados en el punto de descarga al canal de la calle 13 en el municipio de Acacias (Meta).

- En el río Fucha se dispone de la estación limnigráfica El Delirio, localizada $3.5 \mathrm{~km}$ aguas arriba de los vertederos, por lo cual el IDIGER [12] trasladó los caudales hasta el sitio de interés utilizando el método de ajuste probabilístico y transposición de caudales (ver Ecuación (4)), el cual requiere el área de la cuenca perteneciente a la zona de estudio $\left(30.39 \mathrm{~km}^{2}\right)$ y el área de la cuenca correspondiente a la estación $\left(24.73 \mathrm{~km}^{2}\right)$.

$$
Q_{\text {sp }}=\sqrt{\frac{A_{\text {sp }}}{A_{\text {estación }}}} * Q_{\text {estación }}
$$

Donde:

$\mathrm{Q}_{\mathrm{sp}}$ :Caudal en el sitio de proyecto $\left(\mathrm{m}^{3} / \mathrm{s}\right)$

$\mathrm{A}_{\mathrm{sp}}$ : Área de drenaje aferente hasta el sitio de proyecto $\left(\mathrm{km}^{2}\right)$

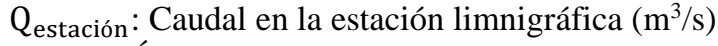

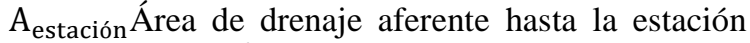
limnigráfica $\left(\mathrm{km}^{2}\right)$

\section{Resultados y discusión}

En todo el rango de caudales simulados del canal escalonado, se obtuvo un flujo tipo saltante (nappe flow) con número de Froude entre 1.0 y 1.7, dando lugar a un resalto ondulante en cada escalón, que se caracteriza por una sucesión de chorros en caída libre que chocan en el siguiente escalón (ver Tabla 2). 
Tabla 2. Tipo de flujo según modelación hidráulica del canal escalonado - Flujo saltante

\begin{tabular}{|c|c|c|c|c|c|}
\hline \multirow[b]{2}{*}{ Escalón } & \multirow[b]{2}{*}{$1.05-0.465 \frac{h}{l}$} & \multicolumn{3}{|c|}{ Yc/h } & \multirow[b]{2}{*}{ Tipo de flujo } \\
\hline & & Caudal máximo & Caudal de diseño & $\begin{array}{l}\text { Caudal de } \\
\text { mínimo }\end{array}$ & \\
\hline 1 & 0.7743 & 0.14920 & 0.10979 & 0.7743 & Saltante \\
\hline 2 & 0.7432 & 0.13661 & 0.10053 & 0.7432 & Saltante \\
\hline 3 & 0.6935 & 0.13185 & 0.09702 & 0.6935 & Saltante \\
\hline 4 & 0.7578 & 0.15323 & 0.11276 & 0.7578 & Saltante \\
\hline 5 & 0.7708 & 0.14174 & 0.10430 & 0.7708 & Saltante \\
\hline 6 & 0.6485 & 0.12063 & 0.08877 & 0.6485 & Saltante \\
\hline 7 & 0.7581 & 0.12599 & 0.09271 & 0.7581 & Saltante \\
\hline 8 & 0.6300 & 0.12599 & 0.09271 & 0.6300 & Saltante \\
\hline 9 & 0.5844 & 0.09144 & 0.06729 & 0.5844 & Saltante \\
\hline 10 & 0.4758 & 0.09071 & 0.06675 & 0.4758 & Saltante \\
\hline 11 & 0.7484 & 0.15533 & 0.11430 & 0.7484 & Saltante \\
\hline 12 & 0.8051 & 0.17445 & 0.12837 & 0.8051 & Saltante \\
\hline 13 & 0.4525 & 0.08722 & 0.06418 & 0.4525 & Saltante \\
\hline 14 & 0.5920 & 0.12599 & 0.09271 & 0.5920 & Saltante \\
\hline 15 & 0.8245 & 0.12599 & 0.09271 & 0.8245 & Saltante \\
\hline
\end{tabular}

Fuente: [9].

Consecuentemente, se observa que al cambiar la rugosidad y material del canal no se afecta su comportamiento hidráulico ni para operación con caudal máximo (ver Figura 7), ni para caudal mínimo (ver Figura 8), indicando que los dos tipos de materiales permiten el desarrollo del flujo saltante en todo el rango de caudales.

Al respecto, en la bibliografía técnica [7] se indica que la transición del flujo saltante a flujo rasante es una función de la altura del escalón y de la pendiente del canal, lo cual ocurre para el régimen de flujo saltante siempre que se cumpla con la Ecuación (5), condición que se demostró en los dos modelos hidráulicos (ver Tabla 2).

$$
\frac{\mathrm{d}_{\mathrm{c}}}{\mathrm{h}}<1.05-0.465 \frac{\mathrm{h}}{\mathrm{l}}
$$

Donde:

$\mathrm{d}_{\mathrm{c}}$ : Profundidad crítica del canal (m)

$\mathrm{h}$ : Altura del escalón (m)

1 : Longitud del escalón (m)

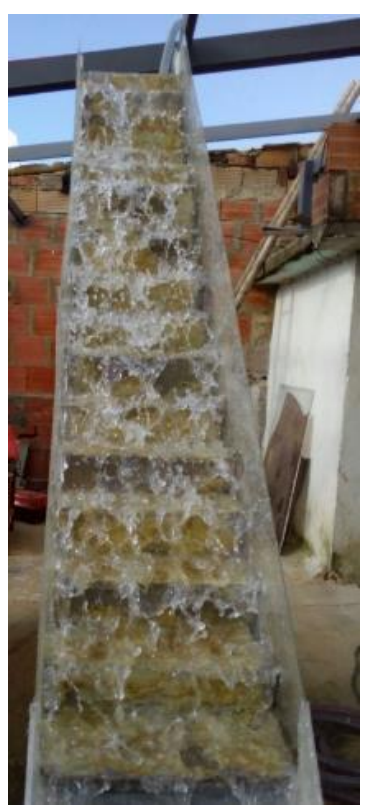

(a)

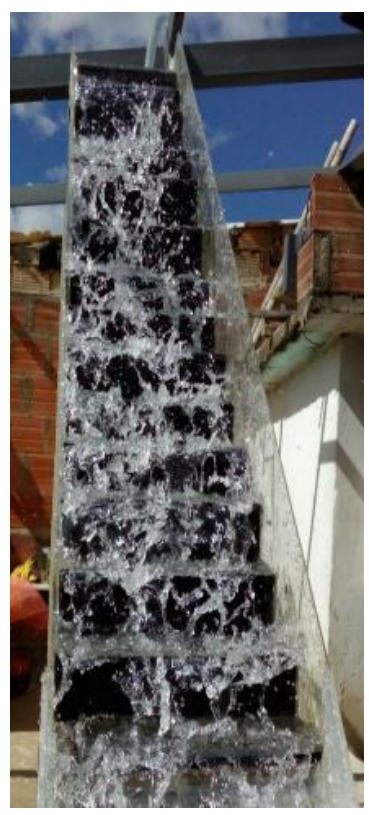

(b)

Figura 7. Régimen de flujo saltante en los dos modelos hidráulicos de canal escalonado (a) en concreto y (b) revestido en neumático usado, para caudal máximo (1.28 1/s). Fuente: [9] 
Evaluación de la eficiencia en disipación de energía en estructuras hidráulicas construidas con gaviones y material reciclado (neumático usado) mediante modelamiento físico a escala reducida

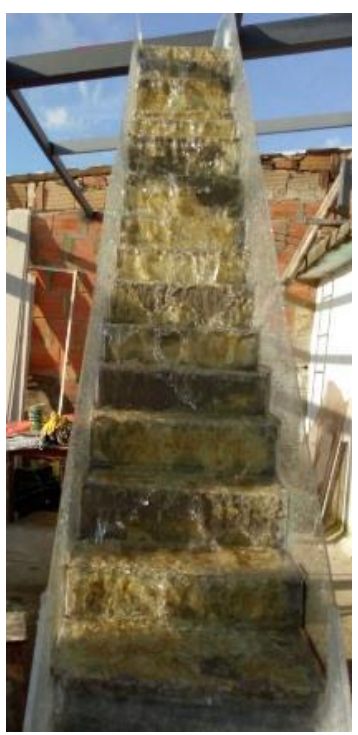

(a)

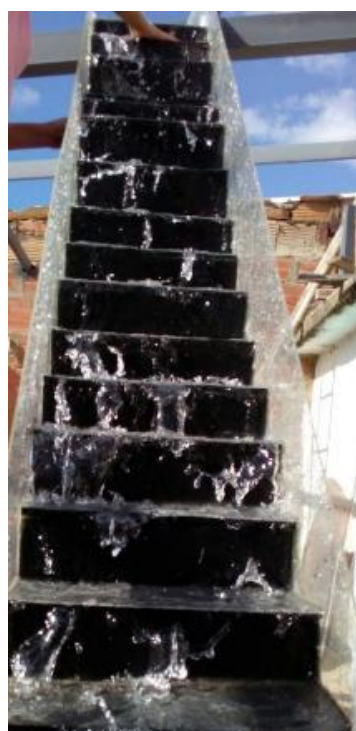

(b)
Figura 8. Régimen de flujo saltante en los dos modelos hidráulicos de canal escalonado (a) en concreto y (b) revestido en neumático usado, para caudal mínimo (0.42 1/s). Fuente: [9]

La eficiencia en reducción de energía específica fue hasta el $2.1 \%$ con el caudal de diseño (ver Tabla 3 ), demostrado que al revestir la estructura con neumático usado, se conserva una disipación de energía similar a la estructura convencional revestida en concreto (ver Figura 9).

Se llama la atención que con caudales mínimos las pérdidas de energía en el modelo arrojan valores negativos, asociadas a la precisión de la instrumentación utilizada, lo cual impide determinar con mayor precisión dicha pérdida.

Sin embargo, el análisis de costos, incluyendo materiales y mano de obra, muestra una reducción del 18.5\%, pasando de $\$ 31.220 .300$ con el canal en concreto a $\$ 25.470 .900$ para el canal en neumático.

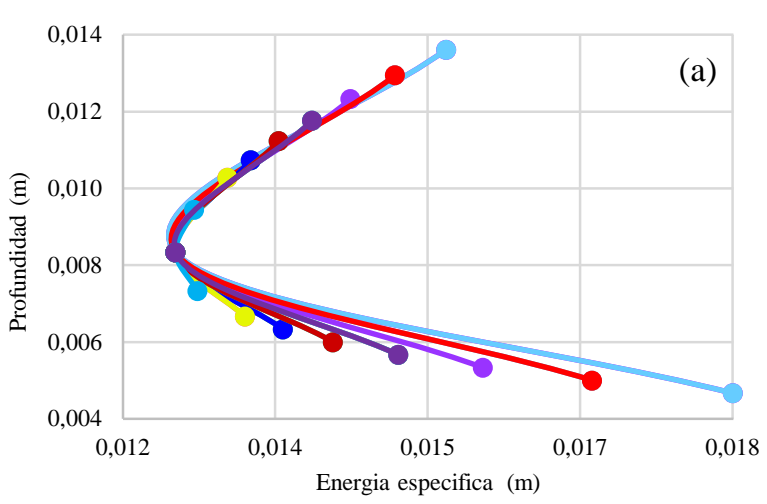

(a)

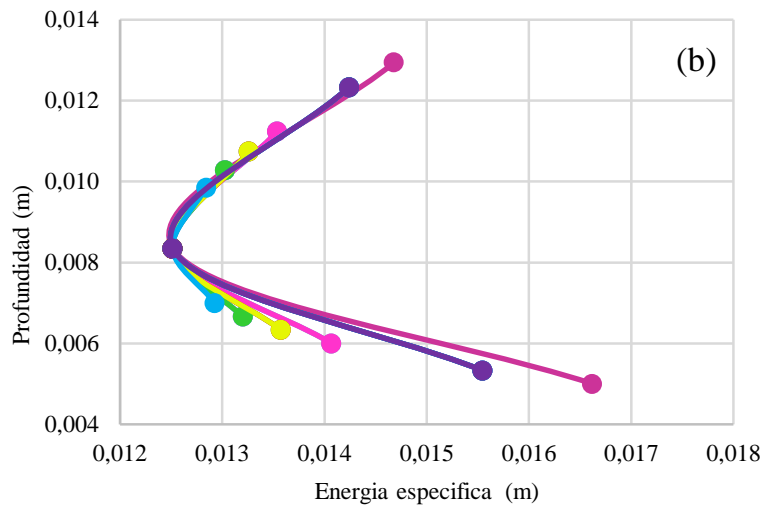

(b)

Figura 9. Curva de energía específica para (a) el canal escalonado en concreto y (b) revestido en neumático usado. Fuente: [9].

En el caso del pozo con estructura de caída, en todos los casos se observó un flujo saltante, lo cual indica según [13], que se simularon únicamente caudales bajos respecto a la capacidad de la estructura, en cuanto, para un caudal pequeño sobre la estructura de caída, el flujo corre de un escalón a otro como una sucesión de cascadas pequeñas, correspondiendo con un flujo saltante, y en la medida que el caudal aumenta se desarrolla un flujo rasante (ver Figura 10).

Tabla 3. Resultados disipación de energía según modelación hidráulica del canal escalonado.

\begin{tabular}{|l|l|l|l|l|l|l|}
\hline \multirow{2}{*}{ Parámetro } & \multicolumn{2}{|l|}{ Caudal máximo } & \multicolumn{2}{l|}{ Caudal de diseño } & \multicolumn{2}{l|}{ Caudal mínimo } \\
\cline { 2 - 7 } & Concreto & Neumático & Concreto & Neumático & Concreto & Neumático \\
\hline Energía específica aguas arriba (m) & 0.3149 & 0.3139 & 0.2234 & 0.2127 & 0.1337 & 0.1365 \\
Energía específica aguas abajo (m) & 0.2878 & 0.2866 & 0.2079 & 0.2029 & 0.1445 & 0.1457 \\
Pérdida de energía (m) & 0.0271 & 0.0273 & 0.0154 & 0.0098 & -0.0108 & -0.0092 \\
Eficiencia (\%) & $\mathbf{9 2 . 0}$ & $\mathbf{9 2 . 2}$ & $\mathbf{9 3 . 6}$ & $\mathbf{9 5 . 7}$ & $\mathbf{1 0 7 . 4}$ & $\mathbf{1 0 6 . 5}$ \\
\hline
\end{tabular}

Fuente: elaboración propia. 


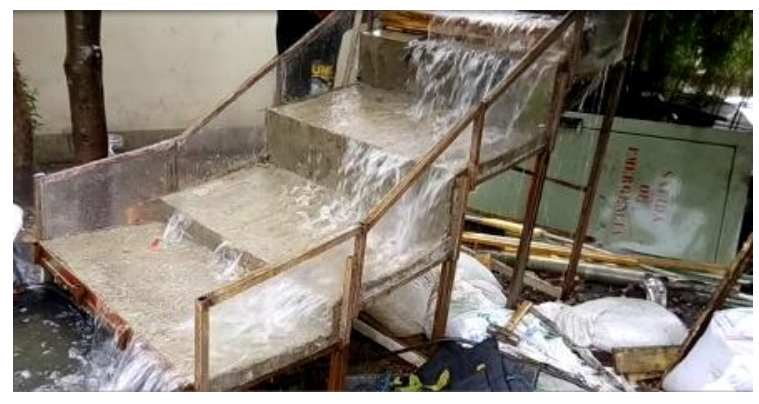

Figura 10. Régimen de flujo saltante en el modelo hidráulico de la estructura de caída con canal escalonado. Fuente: [10].

Los resultados indican que la eficiencia en disipación de energía aumenta con el caudal para revestimiento en concreto, alcanzando valores máximos de $288.5 \%$ y mínimos de hasta $102.7 \%$, mientras que en la estructura con neumático la eficiencia se normaliza entre un $75.4 \%$ y $108.5 \%$, y aunque es inferior a la eficiencia con concreto, es aceptable, en cuanto la energía específica disminuye para caudales altos $(0.022 \mathrm{~m}$ en concreto y $0.014 \mathrm{~m}$ en neumático) y cumple, con lo establecido en la Resolución 0330 de 2017 [6] y el manual del INVIAS [7], según los cuales, las cámaras de caída en los sistemas de alcantarillado son necesarias en zonas de alta pendiente que tienen como función dirigir el flujo, disipar una importante cantidad de energía y proteger la estructura contra impactos en las paredes para mantener estabilidad estructural y evitar socavación (ver Tabla 4).
Así las cosas, la estructura revestida en neumático permite cumplir con las funciones de disipación de energía y protección de la estructura, pero con una reducción significativa de costos y beneficios ambientales, dada la posibilidad de reutilizar llantas usadas.

Con la modelación del río Fucha, los dos tipos de vertederos reducen el potencial erosivo del flujo respecto a la velocidad del río sin estructuras que supera la velocidad permitida para el material del lecho $(1.68 \mathrm{~m} / \mathrm{s})$ caracterizado como una matriz heterogénea de arcillas plásticas de color gris oscuro, con interestratificaciones lenticulares de arena y grava, que hacen estos suelos muy susceptibles a procesos de socavación por la acción de la corriente de agua (ver Figura 11).

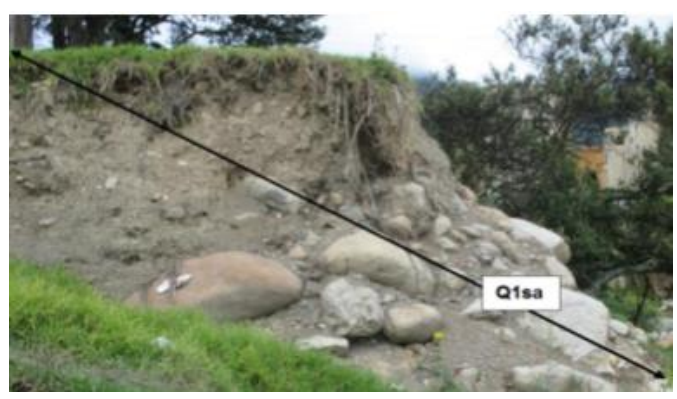

Figura 11. Composición y estructura de los suelos en inmediaciones al río Fucha. Fuente: [11].

Tabla 4. Resultados disipación de energía según modelación hidráulica del pozo con estructura de caída.

\begin{tabular}{|c|l|l|l|l|l|l|}
\hline \multirow{2}{*}{ Parámetro } & \multicolumn{2}{l|}{ Caudal máximo } & \multicolumn{2}{l|}{ Caudal de diseño } & \multicolumn{2}{l|}{ Caudal mínimo } \\
\cline { 2 - 7 } & Concreto & Neumático & Concreto & Neumático & Concreto & Neumático \\
\hline Escalón 1. Energía específica (m) & 0.021 & 0.014 & 0.024 & 0.014 & 0.022 & 0.014 \\
Pérdida de energía (m) & -0.023 & 0.000 & -0.044 & 0.000 & -0.029 & 0.000 \\
Eficiencia (\%) & $\mathbf{2 0 9 . 5}$ & $\mathbf{9 9 . 0}$ & $\mathbf{2 8 8 . 5}$ & $\mathbf{9 6 . 9}$ & $\mathbf{2 3 3 . 2}$ & $\mathbf{9 8 . 1}$ \\
\hline Escalón 2. Energía específica (m) & 0.015 & 0.014 & 0.016 & 0.014 & 0.019 & 0.011 \\
Pérdida de energía (m) & -0.008 & 0.001 & -0.011 & 0.001 & -0.028 & 0.000 \\
Eficiencia (\%) & $\mathbf{1 5 1 . 7}$ & $\mathbf{9 2 . 9}$ & $\mathbf{1 7 1 . 4}$ & $\mathbf{9 2 . 9}$ & $\mathbf{2 5 1 . 7}$ & $\mathbf{9 9 . 9}$ \\
\hline Escalón 3. Energía específica (m) & 0.010 & 0.016 & 0.010 & 0.016 & 0.014 & 0.010 \\
Pérdida de energía (m) & 0.001 & 0.004 & 0.001 & 0.004 & -0.010 & -0.001 \\
Eficiencia (\%) & $\mathbf{1 0 2 . 7}$ & $\mathbf{7 5 . 4}$ & $\mathbf{1 0 2 . 7}$ & $\mathbf{7 5 . 4}$ & $\mathbf{1 7 3 . 2}$ & $\mathbf{1 0 8 . 5}$ \\
\hline
\end{tabular}

Fuente: elaboración propia

No obstante, el análisis de costos unitarios demuestra que, al reemplazar el revestimiento de concreto por neumático usado, el costo de la estructura se reduce en $\$ 20.25$ millones, que representa una reducción del $85.3 \%$ en el costo de la obra (ver Tabla 5).
Sin embargo, para caudales superiores, prevalece el flujo sobre la estructura, de manera que los vertederos en gavión reducen la velocidad del flujo en mayor proporción que las estructuras existentes (ver Figura 12). 
Evaluación de la eficiencia en disipación de energía en estructuras hidráulicas construidas con gaviones y material reciclado (neumático usado) mediante modelamiento físico a escala reducida

Tabla 5. Costos unitarios de materiales y obra civil para construcción de estructura de caída en un pozo

\begin{tabular}{|c|c|c|c|c|c|c|c|}
\hline \multirow[b]{2}{*}{ Item } & \multirow[b]{2}{*}{ Concepto } & \multirow[b]{2}{*}{ Unidad } & \multirow{2}{*}{$\begin{array}{l}\text { Valor } \\
\text { Unitario } \\
\text { (\$ COP) }\end{array}$} & \multicolumn{2}{|l|}{ Concreto } & \multicolumn{2}{|l|}{ Neumático } \\
\hline & & & & Cantidad & $\begin{array}{l}\text { Valor Total } \\
\text { (\$ COP) }\end{array}$ & Cantidad & $\begin{array}{l}\text { Valor Total } \\
\text { (\$ COP) }\end{array}$ \\
\hline 1 & $\begin{array}{l}\text { Suministro, armado y fisurado } \\
\text { de hierro de refuerzo }\end{array}$ & $\mathrm{Kg}$ & 3643 & 2735.14 & 9964115 & & \\
\hline 2 & Armado de formaleta & ML & 10558 & 6.6 & 69683 & & \\
\hline 3 & $\begin{array}{l}\text { Suministro y fundida de } \\
\text { concreto } 4000 \text { PSI }\end{array}$ & M3 & 450454 & 30.27 & 13636143 & & \\
\hline 4 & Desencofrado & M2 & 3185 & 23.76 & 75676 & & \\
\hline 5 & Anclajes para neumático & M2 & 37157 & & & 23.76 & 882850 \\
\hline 6 & $\begin{array}{l}\text { Cargue y transporte de } \\
\text { neumático usado }\end{array}$ & VIAJE & 283058 & & & 1 & 283058 \\
\hline 7 & $\begin{array}{l}\text { Suministro, armado y } \\
\text { figurado de neumático usado }\end{array}$ & M2 & 56015 & & & 23.76 & 1330916 \\
\hline 8 & Sellado de juntas con silicona & Un & 24816 & & & 40 & 992640 \\
\hline & Total & & & & 23745617 & & 3489464 \\
\hline
\end{tabular}

Fuente: [10].

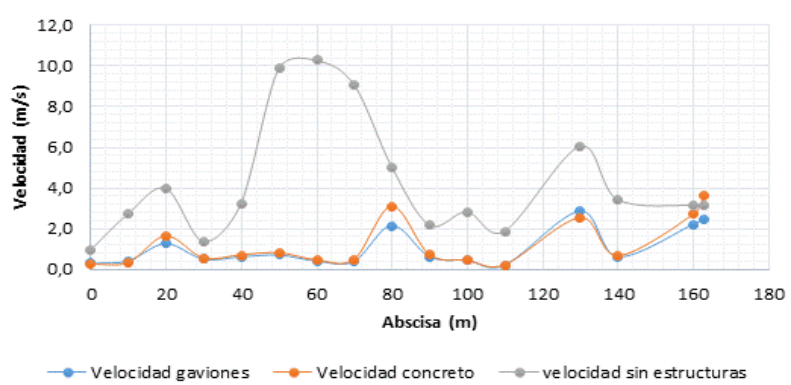

Figura 12. Velocidad del río para las tres configuraciones del modelo físico a caudal de $3.9 \mathrm{~m} / \mathrm{s}$. Fuente: [11].

Puede observarse en la Tabla 6, que la eficiencia de disipación de energía de los vertederos de caída libre en concreto disminuye al aumentar el caudal, mientras que las estructuras en gavión tienden a aumentar la eficiencia desde un caudal de $0.82 \mathrm{~m}^{3} / \mathrm{s}$ a $3.89 \mathrm{~m}^{3} / \mathrm{s}$ debido al flujo rasante que se genera. Sin embargo, para el caudal de $7.38 \mathrm{~m}^{3} / \mathrm{s}$ los vertederos escalonados en gavión no presentaron aumento en la eficiencia de disipación de energía y se puede inferir que alrededor de este caudal la eficiencia tiende a disminuir. Por otro lado, para caudales de 0.82 a $3.89 \mathrm{~m}^{3} / \mathrm{s}$ el potencial de disipación de energía es mayor en los vertederos de caída libre en concreto.

Para el análisis de las líneas de flujo, se aplicó azul de metileno al agua, logrando identificar que, con el cauce natural, sin vertederos, el flujo se concentra sobre el talud izquierdo hasta que se deflecta bruscamente hacia el talud derecho (ver Figura 13), condición que redunda en la socavación de las márgenes del río y potencial afectación de la vía existente y las viviendas aledañas.

Tabla 6. Eficiencias de disipación de energía de las dos estructuras estudiadas en el modelo a escala

\begin{tabular}{|c|c|c|c|c|c|c|c|}
\hline \multirow{2}{*}{ Abscisa } & \multirow{2}{*}{$\mathbf{Q}\left(\mathbf{m}^{3} / \mathbf{s}\right)$} & \multicolumn{3}{|c|}{ Vertedero caída libre en concreto } & \multicolumn{3}{|c|}{ Vertedero escalonado en gavión } \\
\hline & & E1 (m) & E2 (m) & $\% \mathrm{E}$ & E1 (m) & E2 (m) & $\% \mathrm{E}$ \\
\hline $\mathrm{k} 0+11$ & & 1.421 & 0.693 & $51.277 \%$ & 0.921 & 0.856 & $7.071 \%$ \\
\hline $\mathrm{k} 0+70$ & 0.82 & 1.085 & 0.595 & $45.123 \%$ & 0.495 & 0.504 & $-1.809 \%$ \\
\hline $\mathrm{k} 0+110.54$ & & 1.154 & 0.475 & $58.795 \%$ & 0.474 & 0.508 & $-7.238 \%$ \\
\hline k 0+11 & & 1.630 & 0.888 & $45.513 \%$ & 1.337 & 0.927 & $30.667 \%$ \\
\hline $\mathrm{k} 0+70$ & 3.89 & 1.243 & 1.068 & $14.143 \%$ & 1.297 & 0.926 & $28.589 \%$ \\
\hline $\mathrm{k} 0+110.54$ & & 1.235 & 0.921 & $25.389 \%$ & 1.301 & 0.971 & $25.410 \%$ \\
\hline $\mathrm{k} 0+11$ & & 1.731 & 1.158 & $33.070 \%$ & 1.578 & 1.154 & $26.894 \%$ \\
\hline $\mathrm{k} 0+70$ & 7.38 & 1.395 & 1.679 & $-20.326 \%$ & 1.566 & 1.191 & $23.931 \%$ \\
\hline $\mathrm{k} 0+110.54$ & & 1.394 & 1.715 & $-22.990 \%$ & 1.687 & 1.258 & $25.421 \%$ \\
\hline
\end{tabular}

Fuente: elaboración propia. 


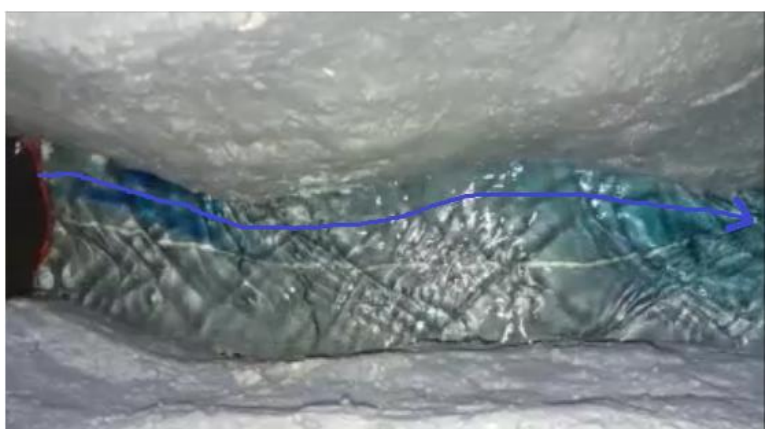

(a)

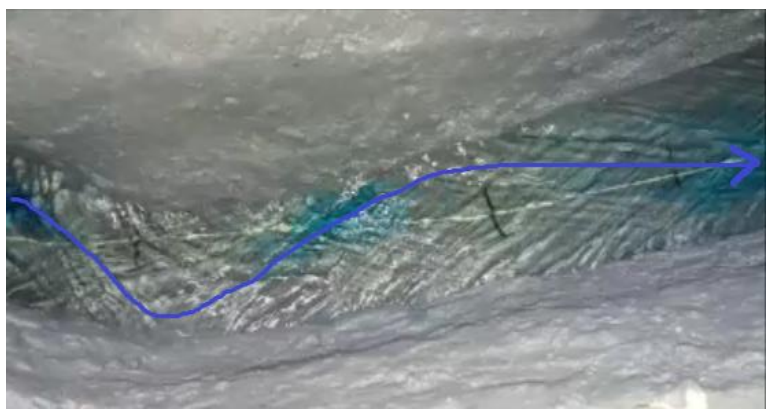

(b)

Figura 13. Líneas de flujo en modelo hidráulico sin estructuras de disipación (a) entre k0+000 y k0+030 y

(b) entre k0+030 y k0+060. Fuente: [11].

Al incluir los vertederos de caída libre, aguas abajo se generan vórtices debido a la turbulencia del resalto (ver Figura 14), ocasionando retención de flotantes y mayor riesgo de socavación de taludes del río y los mismos estribos del vertedero, aunado a ello, el flujo no se desarrolla por el eje del cauce, sino que se desplaza hacia los taludes.

Finalmente, con los vertederos escalonados, hacia aguas arriba de estas estructuras se presentan vórtices de baja velocidad debido al choque del agua con los vertederos, pero hacia aguas abajo la línea de flujo se desarrolla por el eje del río, desaparecen los vórtices que actualmente generan socavación de los taludes y los cambios de dirección se suavizan, pasando de ángulos de deflexión de $45^{\circ}$ a solo $11^{\circ}$, significando menor potencial erosivo (ver Figura 15).

Producto de la concentración del flujo por el eje del río y los ángulos de deflexión más pequeños, el punto de impacto hacia aguas abajo de los vertederos se aumenta en un $75 \%$ respecto a las estructuras de caída libre, es decir, el punto de impacto del agua se desplaza de la abscisa k0+040 a la abscisa k0+070, demostrando un mejor comportamiento hidráulico (ver Figura 16).

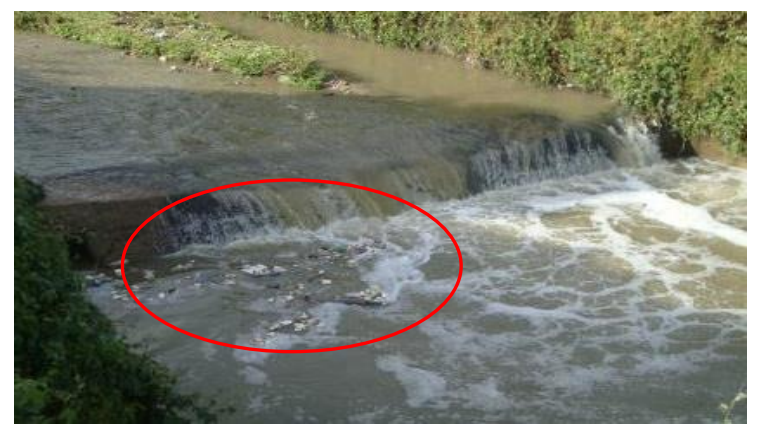

(a)

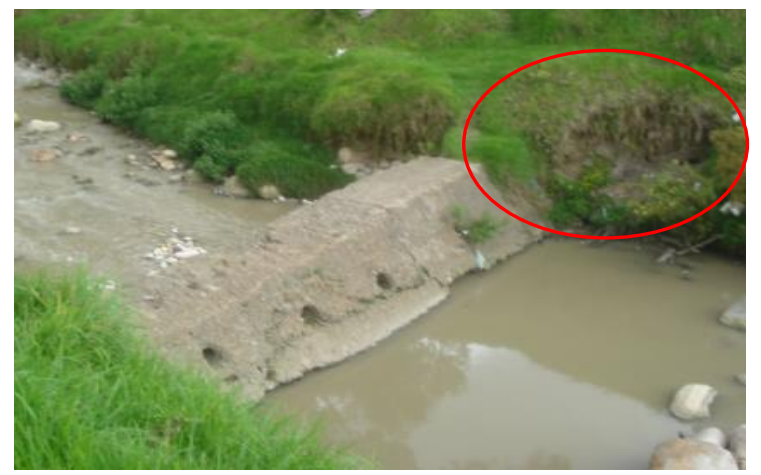

(b)

Figura 14. (a) Vórtices y (b) socavación en taludes localizados aguas abajo de los vertederos de caída libre. Fuente: [11].

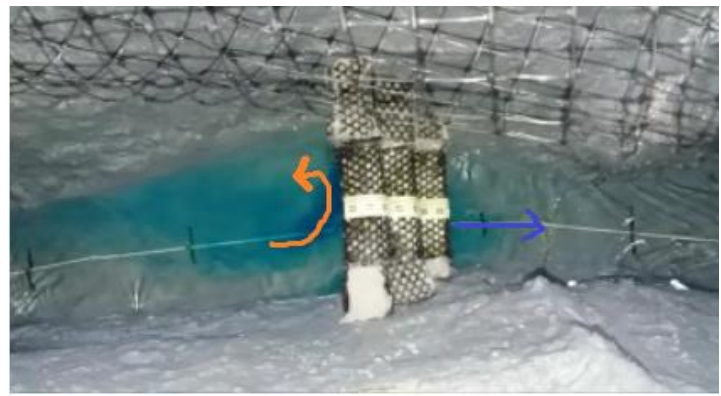

(a)

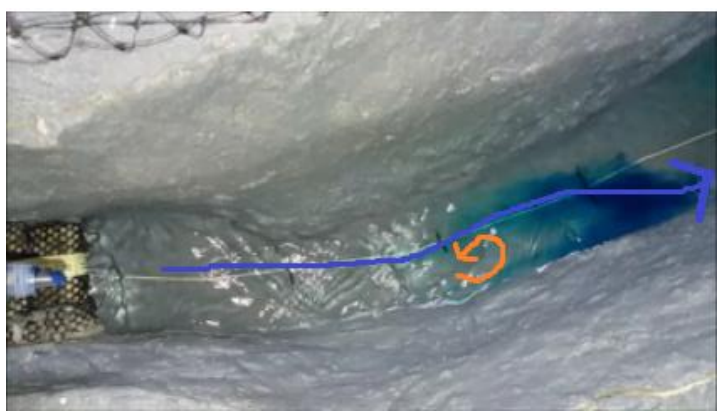

(b)

Figura 15. (a) Líneas de flujo aguas arriba y (b) aguas abajo del vertedero escalonado. Fuente: [11]. 
Evaluación de la eficiencia en disipación de energía en estructuras hidráulicas construidas con gaviones y material reciclado (neumático usado) mediante modelamiento físico a escala reducida

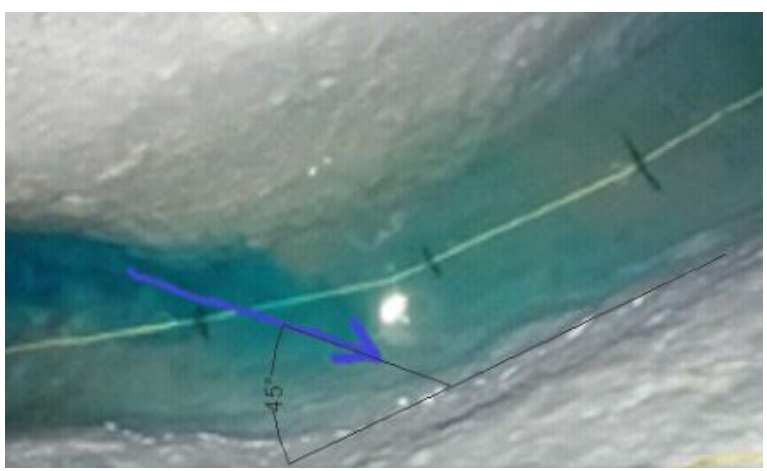

(a)

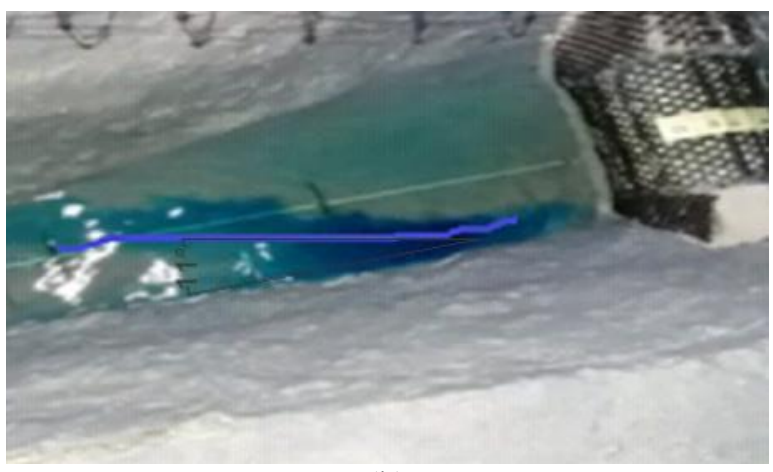

(b)

Figura 16. Ángulo de choque de la línea principal de flujo con el talud derecho del río (a) con vertederos es caída libre, (b) con vertederos escalonados en gavión.

Fuente: [11].

\section{Conclusiones}

Al implementar neumático usado como revestimiento de estructuras hidráulicas tipo canal escalonado y estructuras de caída, se conserva un comportamiento del flujo similar a la estructura revestida en concreto, en términos del tipo de flujo (nappe flow) y una disipación de energía superior al $92 \%$, pero con una reducción en costos del $18.5 \%$ y el aprovechamiento de materiales no biodegradables, obteniendo un impacto ambiental y económico positivo. La eficiencia de disipación de energía de los vertederos en concreto tiende a disminuir a mayor caudal, mientras que en los vertederos escalonados en gavión aumenta la eficiencia, hasta cuando las estructuras de disipación se ahogan.

Los vertederos en este tramo del río Fucha, tienen el propósito de proteger la estabilidad morfológica del terreno y las estructuras aledañas al río, pues los dos tipos de vertederos reducen la velocidad hasta límites no erosionables. Morfológicamente, las líneas de flujo con los vertederos escalonados en gaviones logran eliminar vórtices que aparecen abajo de los vertederos convencionales y concentran el flujo por el eje del cauce, suavizan los cambios de dirección, dando lugar a un flujo más rápido, pero concentrado en la parte central del cauce.

Por tanto, se concluye que el uso gaviones y material reciclado (neumático usado) aporta a preservar la integridad, durabilidad y correcto funcionamiento de las estructuras de disipación de energía, adicional a su bajo costo, bajo impacto ambiental y control morfológico.

\section{Referencias}

[1] L. Ayala, "Modelo experimental para el estudio de la disipación de energía mediante el uso de gaviones en canales homogéneos en el control de inundaciones," Rev. Epsilón, vol. 14, pp. 093-105, 2010.

[2] R. French, Hidraulica de canales abiertos. México: McGraw-Hill, 1988.

[3] V. Chow, Hidraulica de canales abiertos, Bogotá: McGraw-Hill, 2004.

[4] R. Flores, "Flujo en canales abiertos Cátedrade Mecánica de Fluidos," noviembre, 2015. [Presentación en SlideShare]. Disponible en: http://es.slideshare.net/rosemaryflorees/trabajo-fluidos55635115

[5] S. Villamarin, "Manual basico de diseño de estructuras de disipacion de energia Hdraulica," trabajo de fin de grado, Escuela Politécnica del Ejército, 2013.

[6] Reglamento Técnico del Sector de Agua Potable y Saneamiento Básico, Resolución 0330 Ministerio de Vivienda, Ciudad y Territorio, Colombia, 2017.

[7] Manual de drenaje para carreteras Colombia, INVIAS, 2009.

[8] H. Chanson, "Prediction of the transition nappe/skimming flow on a stepped channel", Journal of Hydraulic Research, vol 34:3, pp. 421-429. doi: 10.1080/00221689609498490

[9] M. García y O. Bravo, “Comparación de la eficiencia hidráulica de un canal escalonado revestido en concreto y en material reciclable (neumático usado) a partir de un modelo físico," trabajo de fin de grado, Universidad de La Salle, 2017.

[10] I. Acero y C. Vanegas, "Estudio de la disipación de energía mediante un modelo físico en un pozo de alcantarillado pluvial (escalonado) sobre la calle 13 en 
Acacias - Meta, usando material reciclado (neumático)," trabajo de fin de grado, Universidad de La Salle, 2018.

[11] J. Acuña y E. Plazas, "Análisis comparativo de la disipación de energía y patrones de flujo generados por vertederos escalonados en gavión y vertederos de caída libre en concreto en el río Fucha, en la localidad San Cristóbal," trabajo de fin de grado, Universidad de La Salle, 2018.

[12] "Estudio y diseño de obras para la estabilización de márgenes, control de erosión, socavación, y renaturalización del curso del río Fucha en el tramo comprendido desde la carrera 19 este hasta la carrera 1 este, de la Localidad de San Cristóbal de la ciudad de Bogotá," Instituto Distrital de Gestión de Riesgos y Cambio Climático - IDIGER, 2014.

[13] H. Chanson, Hidráulica del flujo en canales abiertos. Bogotá: MacGraw Hill, 2002. 